\title{
BMJ Open Quality Development and implementation of a standardised emergency department intershift handover tool to improve physician communication
}

\author{
Edmund S H Kwok (D) , ${ }^{1}$ Glenda Clapham, ${ }^{1}$ Shannon White, ${ }^{1}$ Michael Austin, ${ }^{2,3}$ \\ Lisa A Calder ${ }^{2}$
}

To cite: Kwok ESH, Clapham G, White S, et al. Development and implementation of a standardised emergency department intershift handover tool to improve physician communication. BMJ Open Quality 2020;9:e000780. doi:10.1136/ bmjoq-2019-000780

- Additional material is published online only. To view please visit the journal online (http://dx.doi.org/10.1136/ bmjoq-2019-000780).

For 'Presented at statement' see end of article.

Received 23 July 2019 Revised 3 January 2020 Accepted 14 January 2020

Check for updates

(C) Author(s) (or their employer(s)) 2020. Re-use permitted under CC BY-NC. No commercial re-use. See rights and permissions. Published by BMJ.

For numbered affiliations see end of article.

Correspondence to Dr Edmund S H Kwok; ekwok@toh.ca

\section{ABSTRACT}

Background Structured handover can reduce communication breakdowns and potential medical errors. In our emergency department (ED) we identified a safety risk due to variation in quality and content of overnight handovers between physicians.

Aim Our goal was to develop and implement a standardised ED-specific handover tool using quality improvement (Ql) methodology. We aimed to increase the proportion of patients having adequate handover information conveyed at overnight shift change from a baseline of $50 \%-75 \%$ in 4 months.

Methods We used published best practices, stakeholder input and local data to develop a tool customised for intershift ED handovers. Implementation methods included education, cognitive aids, policy change and plan-do-studyact cycles informed by end-user feedback. We monitored progress using direct observation convenience sampling. Measures Our outcome measure was proportion of adequate patient handovers (defined as $>50 \%$ of handover components communicated per patient) per overnight handover session. Tool utilisation characteristics were used for process measurement, and time metrics for balancing measures. We report changes using statistical process control charts and descriptive statistics.

Results We observed 49 overnight handover sessions from 2017 to 2019 , evaluating handovers of 850 patients. Our improvement target was met in 10 months (median $=76.1 \%$ ) and proportion of adequate handovers continued to improve to median $=83.0 \%$ at the postimprovement audit. Written communication of handover information increased from a median of $19.2 \%$ to $68.7 \%$. Handover time increased by median $=31 \mathrm{~s}$ per patient. End-users subjectively reported improved communication quality and value for resident education. Conclusions We achieved sustained improvements in the amount of information communicated during physician ED handovers using established QI methodologies. Engaging stakeholders in handover tool customisation for local context was an important success factor. We believe this approach can be easily adopted by any ED.

\section{INTRODUCTION}

\section{Problem description}

Handover in the emergency department (ED) has been highlighted in the literature as a high-risk area for medical errors to occur. ${ }^{1-3}$
Exchange of clinical information between physicians during the transfer of responsibility between shifts has been recognised as problematic. ${ }^{45}$ A prospective observational study in three large metropolitan EDs found that crucial information on management details, investigations and disposition, was perceived to be lacking in $15.4 \%$ of intershift handovers. ${ }^{3}$ A survey of emergency medicine (EM) residency programme directors and trainees in USA revealed large variation in intershift handover practices, prompting a call for standardised processes. ${ }^{6}$ In our own department, we identified a recurring theme in our Morbidity \& Mortality rounds of communication deficiencies during intershift handovers. At the study outset, no standard format for physician-to-physician handover between shifts was being used in our ED. Previous resident research within our department confirmed that patients admitted to inpatient care or referred to a consultant service ('boarded patients') but not yet seen were frequently omitted from intershift handover communication between ED physicians. ${ }^{78}$ Additionally, informal observations of handovers leading up to this quality improvement (QI) initiative revealed that up to $25 \%$ of patients still in active care of the ED team (eg, pending discharge or transportation) were often omitted from handovers between ED physicians, and up to $50 \%$ of patients referred to specialty consultants were omitted from these handovers.

\section{Available knowledge}

Significant benefits in patient safety, communication and daily work have been reported from handover standardisation in inpatient settings, especially those involving multiple professionals and combining verbal and written communication ${ }^{9-11}$ Errors and omissions leading to duplication of efforts and 
delays in patient throughput have been attributed to structure of the handover process. ${ }^{12}$ While handover between the ED and other units (eg, pre-hospital and/ or inpatient) has been well studied, ${ }^{13-17}$ there is a dearth of research pertaining to handover within the ED where care plans initiated by one ED physician are completed by another. Several theoretical frameworks for ED physicianto-physician handover, such as the Areas and Allocation, Beds/Bugs, Colleagues/Consultant, Deaths/Disasaters/ Deserters, Equipment/External Events (ABCDE), have been proposed, ${ }^{18-20}$ and before-after reports have been published on various individualised tools to support handover in the ED (eg, custom checklists, forms and cognitive aids), ${ }^{21-25}$ but none have been robustly validated in practice. Thus, while general principles of intershift handover are described, there is no single accepted framework that is easily applicable to the ED.

\section{Rationale}

We hypothesised that adopting a standardised, systematic approach for handover at shift change would improve the communication of crucial information necessary to seamlessly take over patient cases, prioritise work and be aware of any potential complications that could arise. We postulated that developing a handover tool that suited the nuances of the ED environment, assimilated published learnings and incorporated local physician input would enhance adoption.

\section{Specific aims}

Our aim was to develop and implement an ED-specific handover tool that would ensure communication of the information crucial for care continuity at overnight shift change. Once the handover tool and its discrete information components were defined by stakeholders, we aimed to increase the proportion of patients having at least half of those handover components conveyed at overnight shift change from a baseline of $50 \%-75 \%$ of patients in 4 months.

\section{METHODS}

\section{Context}

\section{The Ottawa Hospital}

At the time of this study, The Ottawa Hospital (TOH) was an 1122-bed academic tertiary care hospital, with the ED receiving over 170000 visits per year. The standard referral practice for ED patients awaiting a consultant assessment and/or admission was for patients to physically remain within the ED until either an inpatient bed became available or a safe discharge process had been implemented.

\section{Department of Emergency Medicine}

The Department of Emergency Medicine (DEM) at $\mathrm{TOH}$ is responsible for providing emergency physician coverage at two campuses and providing accredited EM training for Royal College of Physicians of Canada and
Canadian College of Family Physicians-Emergency Medicine. During the study period (Spring 2016-Summer 2018), there were approximately 70 staff EM physicians and 59 EM trainees working in the DEM. Numerous offservice residents and medical students also regularly do clinical rotations through the department.

\section{ED shift structure}

At the time of this study, physician staffing hours for the ED were matched to patient arrival patterns, with the total number of staff EM physicians progressively escalating through the day and tapering off towards the end of the evening. The lowest rate of patient arrivals occurs after midnight, so the shift schedule allocates a single overnight staff EM physician (24:00-08:00) at each of the two ED campuses. This shift structure creates a situation in which multiple evening physicians (potentially up to four different staff physicians and their associated learners) communicate their patient handovers to a single overnight EM physician. Majority of handover communication is done directly between staff physicians; however senior EM residents often perform this task with direct supervision of the responsible staff physician as part of their training and progression of competencies.

\section{Health records and electronic patient tracking board}

At the time of this study, ED patient visits were documented on paper health records and subsequently scanned into an electronic database, augmented by an interactive electronic patient tracking system ('whiteboard') and computerised physician order entry system (CPOE) for diagnostic imaging. The whiteboard has an electronic note feature ('sticky note') which allows physicians to enter simple free-text notes on each ED patient. Once saved, electronic sticky notes are accessible to all ED team members, including nursing and other allied health professionals, until the patient is discharged from the ED.

\section{Intervention}

Development of handover tool

We performed a literature review of published articles describing handover practices, that focused on ED-specific models and experiences with demonstrated positive quality and safety improvements. ${ }^{1} 3478101214$ 16-18 20-23 25 There is no gold standard that is currently accepted to define 'adequate' ED intershift handover. Local quality improvement and patient safety experts summarised key learnings from the literature and local observational analysis, ${ }^{78}$ and used them as a framework for leading focus groups in our department.

We invited members of our DEM physician, resident, nursing and allied health professional team to participate in focus groups. The lead author facilitated three 1-hour focus group sessions (five to six participants each, with three to four staff physicians, one resident physician and one allied health professional) using a structured agenda: introductions, background on our current ED handover 
Table 1 Thematic categories from focus groups

\begin{tabular}{|c|c|c|}
\hline Thematic category & Description & Acronym key \\
\hline Patient identification & $\begin{array}{l}\text { Name, age, gender } \\
\text { Location within the department } \\
\text { Relevant patient medical history } \\
\text { Mode of arrival (eg, ambulance, family, self, police) }\end{array}$ & Entity \\
\hline Chief complaint & $\begin{array}{l}\text { Working diagnosis } \\
\text { Primary complaint/issue }\end{array}$ & Diagnosis \\
\hline Investigations & Investigations ordered/completed/awaiting results & Investigations \\
\hline Care/treatment orders & $\begin{array}{l}\text { Medications that have been given (home medications, emergent medications) } \\
\text { Orders that have been given } \\
\text { (fluids, nursing orders, etc) }\end{array}$ & Treatments \\
\hline Constraints to disposition & $\begin{array}{l}\text { Patient home situation (supports, etc) } \\
\text { Documents / forms completed or required to facilitate disposition } \\
\text { Communication with family, other care providers }\end{array}$ & Logistics \\
\hline Services & $\begin{array}{l}\text { Specialty services involved in patient care } \\
\text { Status of consultant requests }\end{array}$ & Services \\
\hline
\end{tabular}

processes, a summary of published literature available and open discussion and feedback anchored by core probing questions: 'What are the biggest quality gaps in our current handovers?'; 'What works well in our current handovers?'; and 'What are crucial components of an ideal ED handover in our department?'. Key findings were recorded by the facilitator throughout the sessions and later analysed for themes.

Taken together with the results of our literature review and local observational analyses, the findings from the focus groups were summarised into discrete categories of handover information specific to our ED environment, represented by the acronym ED-VITALS (table 1). Focus group participants recommended separating the tool to address two unique ED patient populations: those whose most responsible physician (MRP) is the EM physician overseeing the department (EM MRP), and those who are admitted and/or referred to consultant services but still physically located in the ED (non-EM MRP). Hence, the ED-VITALS acronym was split into: ED-VITAL for patients with an EM MRP; and ED-VSA for patients admitted and/ or referred with a non-EM MRP (online supplementary appendix 1a) We presented the proposed model to our staff physicians during a monthly departmental meeting for face validation and final feedback prior to testing and implementation.

Intended utilisation of handover tool as a framework

There was universal feedback during the focus group sessions that our local physicians would oppose any process change that added new paper forms or checklists to our existing complex paper health record process. We were also aware of evidence that suggested complex processes, such as handover of an entire population of ED patients to another physician, require more than a simple checklist. ${ }^{9} 11151622$ 26-28 Thus, our handover tool is intended to provide a framework that physicians can adopt to prepare for, organise, deliver, receive and follow-up on handovers, rather than an in-the-moment checklist to be completed at the point of shift change. We emphasised this approach to the handover tool during the education sessions (see below) and it was reinforced by the free-text documentation format on our electronic patient tracking system.

\section{Implementation plan}

Between the spring of 2017 and summer of 2018, we implemented our new handover tool using a multifaceted approach with educational, structural and procedural components. In the winter of 2019 we performed a postimplementation audit to check for sustainability.

\section{PDSA-1}

The first of three PDSA cycles began in June 2017 after focus group findings and the proposed handover tool were presented at a DEM physician department meeting. That meeting was also used to reinforce how the new handover tool could be used to prepare and deliver a concise handover. In September, EM residents were provided with formal education during a scheduled academic session. We followed up each presentation with 
email communication summarising the key information of the new handover process.

We supplemented the educational components with two types of cognitive aids: table top posters summarising the handover tool, placed at all designated handover locations (online supplementary appendix 2); and pocket cards, sized to be easily carried around on shift, distributed to all staff physicians and placed at each handover station.

We also strategically recruited DEM staff physicians who preferentially worked more overnight shifts than others and developed them as champions for this new handover process. As the oncoming night physician receiving handovers, these individuals were well positioned to reinforce any changes implemented and fuel frontline engagement throughout the project.

Our QI coordinator attended a random sample of overnight handover sessions and solicited informal survey feedback from physician and resident end-users. Physicians who received handover were invited to provide their reflections on the usefulness of the handover communication at the end of their shift, through written surveys.

\section{PDSA-2}

The feedback received during PDSA-1 was used in October 2017 to modify the format of the handover tool. Most of the changes were relatively minor, including simplifying and streamlining the amount of information contained within the pocket cards. The revised pocket card (online supplementary appendix 1b) was first shown to three different frontline physicians for face validity, before being introduced to staff and resident physicians in November through a newsletter and departmental presentations, which included findings to date and tips for effective handovers. Reminder emails were sent to all DEM members to encourage adoption of the new handover tool. Observations and informal surveys were conducted in the same format as for PDSA-1.

\section{PDSA-3}

In March of 2018, a hospital-wide policy was enacted that explicitly defined the ED physician as MRP for all patients physically located in the ED until they were discharged, admitted or seen by a consultant physician. Previously, there was a lack of clarity on when the MRP shifted from EM to non-EM: some members of the EM physician group had worked under the assumption that the transfer of MRP occurred when the consultation request was submitted. The new policy was communicated to all physicians, and we took this opportunity to further emphasise the importance of adequate intershift handovers in our ED.

In addition, we incorporated recurring ED handover training during regularly-scheduled departmental continuing professional development (CPD) sessions for ED staff physicians. The first CPD handover session occurred in June 2018, with repeating sessions planned every 6-12 months. As incentives, these sessions were accredited to provide Continuing Medical Education (CME) credits as part of local certification CPD requirements.

\section{Study of intervention}

A QI coordinator (GC) performed direct observations of overnight handovers at both ED campuses using convenience sampling. We decided to only observe overnight handovers for a number of reasons: (a) of all the different ED shift changes in a 24-hour day, overnight shift change typically involved the greatest number of patients being handed off, (b) the rest of the hospital and services are reduced to minimal personnel and resource availability, which theoretically may contribute to higher risk patients being handed over in the ED, and (c) for practicality as we had limited study resources to directly observe handover and thus had to focus on observing the most high yield shift changes. Observations were conducted at baseline (before the handover tool was introduced), during each of the three PDSA cycles and at 6 months postimprovement. A minimum of 10 shift change observations were performed per cycle, and attempts were made to minimise the number of times the same DEM physician's handover was observed. For the direct observations, the QI coordinator arrived unannounced 10-15 min before the intended shift change to gather current state data of the department (eg, total number of eligible patients physically in the ED, number of patients referred to consultants, etc). During the handover communication at shift change, the QI coordinator observed and recorded relevant study data directly into a Microsoft Excel spreadsheet using a tablet computer. The coordinator recorded the start and end time of the handover, the components discussed, presence of an electronic sticky note and patient category. EM physicians were reassured that any data collected were anonymised and their individual performance was not being evaluated.

\section{Measures}

\section{Outcome and process measures}

Our main goal was to improve communication of crucial information at overnight handover, when departmental oversight was assumed by the overnight EM physician. Expert opinion of the focus group participants was that not every patient requires communication of all ED-VITALS components for a seamless transition of care. For example, some ED patients may have very simple ailments that only require a single pending laboratory result for safe discharge, while other patients may be medically complex and require more detailed handover communication. The study investigators agreed that this should average out over the entire ED population, and the consensus was to define a handover as 'adequate' when at least $50 \%$ of the handover components in the corresponding ED-VITALS acronym was verbally communicated and/or a sticky note was written. Therefore, our main outcome measure was defined as the percentage of patients being handed over during an overnight shift 
change who had at least half of the handover components communicated. We subdivided adequacy measure into patient categories (ie, EM MRP, vs non-EM MRP). We gathered qualitative feedback from the physicians receiving handover on the perceived value of handover information. Process measures included how often each component of the handover tool was conveyed by physicians during handover communication and utilisation of the electronic sticky note.

\section{Balancing measures}

Recognising that longer per-patient handover duration has been cited as a contributing factor to errors and omissions, ${ }^{29}$ and that a major concern from EM physicians prior to implementation was the effect of the new tool on handover duration, our main balancing measure was the median duration per patient for verbal handovers. This was calculated as the total duration of the handover divided by the number of patients for whom there was verbal communication during handover. Total duration of the handover session was used as a surrogate marker for impact on department work, recognising that if the total proportion of patients discussed increased, total duration could also increase. Finally, feedback from end-users on tool usefulness in preparing and delivering handover was assessed as a balancing measure.

\section{Analysis}

We used the Excel plugin 'QI Macros' (qimacros.com) to plot statistical process control (SPC) charts to demonstrate changes over time, and descriptive statistics for all measures to quantify change. Process changes associated with each PDSA cycle were identified and annotated in the SPC charts. We re-performed stability analysis after each process change as displayed by stair step control limits on each SPC chart. Adoption rates were assessed by measuring the change in number of tool components used in verbal handovers over time.

\section{RESULTS}

We assessed handovers on 850 individual patients, during 49 overnight shift changes (mean $=15, \mathrm{SD}=4$ per session) across baseline, three PDSAs, and postimprovement audit, representing $76.2 \%$ of the total eligible patients (table 2). The baseline period consisted of seven observation sessions during May 2017; three PDSA cycles consisted of 12,10 and 10 observation sessions respectively between August 2017 and May 2018; and postimprovement audit consisted of 10 observation sessions from January to April 2019. Throughout the study period, most eligible patients were referred/admitted patients with a non-EM MRP (baseline: 66.3\%; improvement: 65.6\%; postimprovement: $64.5 \%$ ). The number of unique staff ED physicians observed doing handovers during the PDSA and audit cycles were 28, 24, 24 and 27 respectively. Residents led handover with direct staff supervision $3 \%, 11 \%, 26 \%$ and $12 \%$ of the time in PDSA-1, PDSA-2, PDSA-3 and final postimplementation audit period, respectively.
Table 2 Patient distribution

\begin{tabular}{|c|c|c|c|}
\hline & $\begin{array}{l}\text { Eligible } \\
\text { patients } \\
\text { physically } \\
\text { in ED at } \\
\text { time of } \\
\text { handover } \\
\text { (n) }\end{array}$ & $\begin{array}{l}\text { Patients } \\
\text { included } \\
\text { in } \\
\text { handover } \\
\text { (n) }\end{array}$ & $\begin{array}{l}\% \text { of } \\
\text { eligible } \\
\text { Patients } \\
\text { included } \\
\text { in } \\
\text { handover } \\
\text { (verbal; } \\
\text { written) }\end{array}$ \\
\hline \multicolumn{4}{|l|}{ Pre-improvement (baseline) } \\
\hline Active ED are (EM MRP) & 57 & 42 & 73.7 \\
\hline $\begin{array}{l}\text { Referred/admitted (non-EM } \\
\text { MRP) }\end{array}$ & 112 & 51 & 45.5 \\
\hline Sub-total & 169 & 93 & 55.0 \\
\hline \multicolumn{4}{|l|}{ Process improvement } \\
\hline \multicolumn{4}{|l|}{ PDSA-1 } \\
\hline Active ED care (EM MRP) & 99 & 83 & 83.8 \\
\hline $\begin{array}{l}\text { Referred/admitted (non-EM } \\
\text { MRP) }\end{array}$ & 159 & 107 & 67.3 \\
\hline Subtotal & 258 & 190 & 73.6 \\
\hline \multicolumn{4}{|l|}{ PDSA-2 } \\
\hline Active ED are (EM MRP) & 79 & 79 & 100.0 \\
\hline $\begin{array}{l}\text { Referred/admitted (non-EM } \\
\text { MRP) }\end{array}$ & 159 & 116 & 73.0 \\
\hline Subtotal & 238 & 195 & 81.9 \\
\hline \multicolumn{4}{|l|}{ PDSA-3 } \\
\hline Active ED are (EM MRP) & 68 & 67 & 98.5 \\
\hline $\begin{array}{l}\text { Referred/admitted (non-EM } \\
\text { MRP) }\end{array}$ & 152 & 115 & 75.7 \\
\hline Subtotal & 220 & 182 & 82.7 \\
\hline \multicolumn{4}{|c|}{ Postimprovement (sustainability audit) } \\
\hline Active ED care (EM MRP) & 82 & 77 & 93.9 \\
\hline $\begin{array}{l}\text { Referred/admitted (non-EM } \\
\text { MRP) }\end{array}$ & 149 & 113 & 75.8 \\
\hline Subtotal & 231 & 190 & 82.3 \\
\hline Total patients & 1116 & 850 & 76.2 \\
\hline
\end{tabular}

ED, emergency department; EM, emergency medicine; PDSA, plando-study-act.

Overall, improvement was seen in the proportion of eligible patients included in the handover and adequacy of handover. Proportion of eligible patients included in handover increased from baseline of $55.0 \%-79.2 \%$ during the improvement process and was measured as $82.3 \%$ in the postimprovement audit (table 2). In the same periods, adequacy improved from baseline median of $50.0 \%$ to $76.1 \%$ to $83.0 \%$ respectively (mean of $45.0 \%$ to $77.1 \%$ to $82.8 \%$ respectively). Figure 1 illustrates the results of our SPC assessment of the adequacy for the two subgroups and all patients for observation sessions at overnight ED shift change. For the subgroup of patients in active care (EM MRP) the median proportion of adequate handovers surpassed the target of $75 \%$ during the first PDSA, and subsequently reached $100 \%$ (mean of $94 \%$ ). For the subgroup of patients who were referred or admitted and under the care of other consulting services 


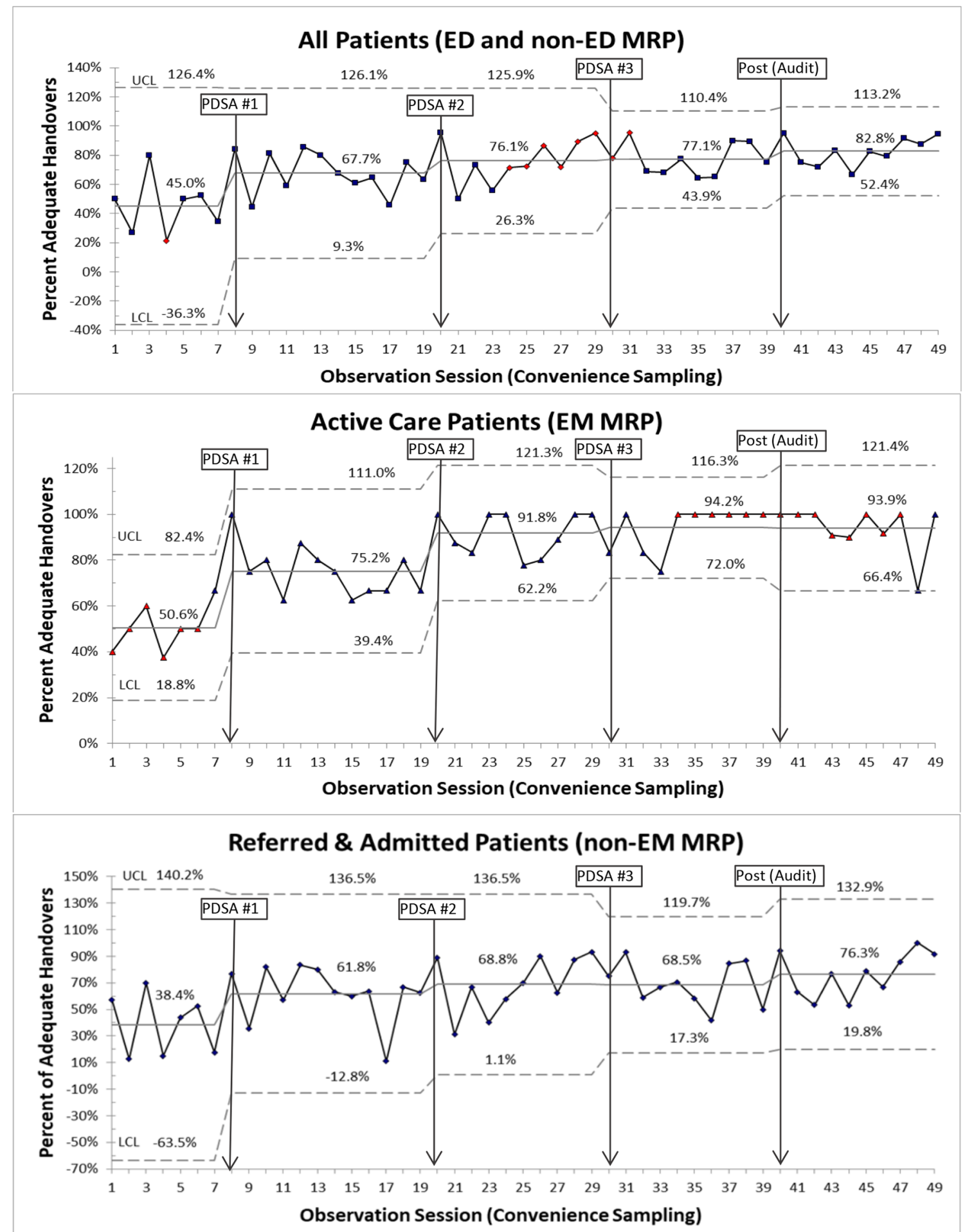

Figure 1 Percentage of patients for whom an adequate amount of information was communicated during handover at overnight shift change. Adequate handover defined as verbally communicating at least $50 \%$ of ED- VITALS components or documentation in electronic note. ED, emergency department; EM, emergency medicine; PDSA, plan-do-study-act.

(non-EM MRP), the median proportion of adequate handovers improved from $43.8 \%$ to $68.6 \%$, and eventually reached $77.9 \%$ in the postimplementation period (means of $38.4 \%, 68.5 \%$, and $76.3 \%$ respectively). The greatest magnitude of improvement occurred in the first PDSA cycle when the new tool was introduced, and extensive teaching occurred; smaller incremental improvements when the tool was modified, when the policy defining MRP was revised, and then after continued use. All improvements were sustained throughout the 13-month study period and beyond.

In the group of patients under active ED care, we observed increased verbal communication of all the different individual ED-VITALS components of the tool 
(table 3). In the postimplementation audit phase, $100 \%$ of patients handed over were explicitly identified (E) with a working diagnosis (D) communicated. Information around Logistics (eg, home living situation, ability to cope at home, regular medications to be dispensed during ED stay, etc) being communicated made the biggest improvement from $10.0 \%$ of patients to $83.9 \%$. Written documentation of handover information using the electronic sticky note increased from $50.0 \%$ to $70.8 \%$.

In the group of patients who have been referred or admitted to a consultant service, information around clinical stability (V) and concrete to-do action (A) items were verbally communicated more frequently after our intervention, while the other components decreased in frequency (table 3). The utilisation of the electronic sticky notes to document written handover information increased from $11.8 \%$ to $66.7 \%$ postimplementation.

The balancing measures of handover duration are depicted in figure 2 with times per session and means for each phase. During the improvement period, total handover duration increased by $6 \min 29 \mathrm{~s}(44 \%)$, and average per-patient time increased by $8 \mathrm{~s}$ (1:08 to 1:16). The median increase was $15 \mathrm{~s}$. In the postimprovement audit period the mean time allocated to each patient was 1:33-a $25 \mathrm{~s}(37 \%)$ increase from baseline, with a median increase of $31 \mathrm{~s}$. Our QI coordinator also noted during observations that the characteristics of the verbal handover changed over time. There was a shift to fewer non-EM MRP patients in the verbal handovers and the exchange changed from didactic-based to more discussion-based on more complex patients.

Qualitative feedback from end users was positive overall, and constructive feedback was used to inform PDSA changes throughout the study period. The tool modifications at the end of PDSA- 1 included simplifications by reducing the amount of text and combining the acronyms into a single format. Some of the common themes and examples of end user feedback are summarised in online supplementary appendix 3. Physicians reported that the tool was helpful in preparation for delivery of handover and creation of sticky notes, by providing a structured format to organise handover communication, and ensured inclusion of often-missed components. Staff and residents reported that the new tool improved both the teaching and learning experience of handover.

\section{DISCUSSION}

\section{Summary of results}

This study describes an ED handover tool implementation using formal QI methodology in an adult population. We demonstrated a considerable improvement in communication of crucial information at ED physician intershift handover during night shift changes through the development and implementation of a structured handover tool. Specifically, we enabled a systemic change in our physician group that resulted in more thorough patient handovers. Crucial information about ED patients, as defined by our own local physicians, were more commonly addressed during handover after our implementation; this improvement continued and was sustained over the course of the 1-year study period and beyond. The impact appeared to be the greatest in the group of patients under active care of the ED team, which intuitively makes sense. Although there appeared to be less explicit verbal communication of individual handover components for patients referred/admitted to consultant services, it appears the information was at least more consistently communicated in written documentation. Despite the increase in useful handover information passed on for each patient, the actual time commitment to do so was relatively minimal with an overall increase in total handover time of less than $7 \mathrm{~min}$. We believe this is a very reasonable trade-off in an 8 hour ED shift, for the significant improvement in the amount of crucial handover information communicated. Both staff and resident physicians commented on the usefulness of having a structured framework as a teaching opportunity around handover practices. It is interesting to note that although we only had the resources to directly observe overnight shift changes, we did not limit the implementation of the framework to those shift changes. Informally we have observed similar uptake in the new handover framework across all shift changes throughout the 24-hour day.

\section{Interpretation}

Several factors likely contributed to the success of this implementation. First, we involved frontline stakeholders in the design of the handover tool. While adopting a previously published handover bundle would have saved development time, the diverse preferences and local culture of our physicians steered us away from a 'plugand-play' solution. Gopwani et al reported a similar positive experience with a locally developed handoff structure and implementation within a paediatric ED. ${ }^{30}$ Our decision was reinforced by the findings of Heilman et al, who explored the suitability of applying the illness severity, patient summary, action list, situational awareness, synthesis (I-PASS) mnemonic in an ED environment, and found that many modifications would be necessary to address the uniqueness of the ED population. ${ }^{31}$ Kicken et al concluded that the idea of completely standardising handover across different stakeholder groups was not realistic given clear differences in preferences of individual groups, and handover improvements necessary to account for physician specialty, level of training and other interprofessional groups such as nursing. ${ }^{32}$ Bearing these observations in mind, we co-designed a customised tool with end-users to enhance adoption and acceptability within our group.

Second, we approached the intervention as a handover 'approach' rather than a 'checklist'. Education alone has been shown to have limited impact on ED handover improvements. ${ }^{33}$ Although some small prestudies and poststudies have shown increased information transfer using a simple checklist, ${ }^{23}{ }^{25}$ others have shown that 


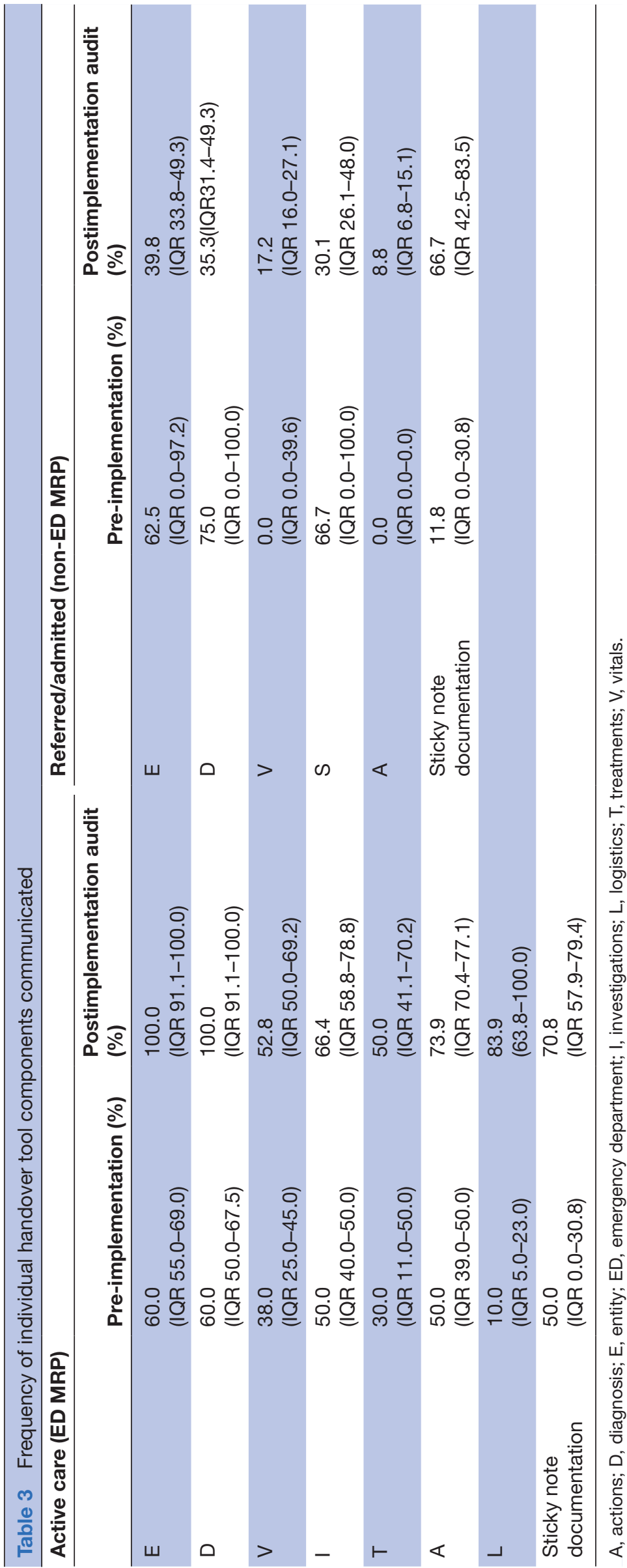




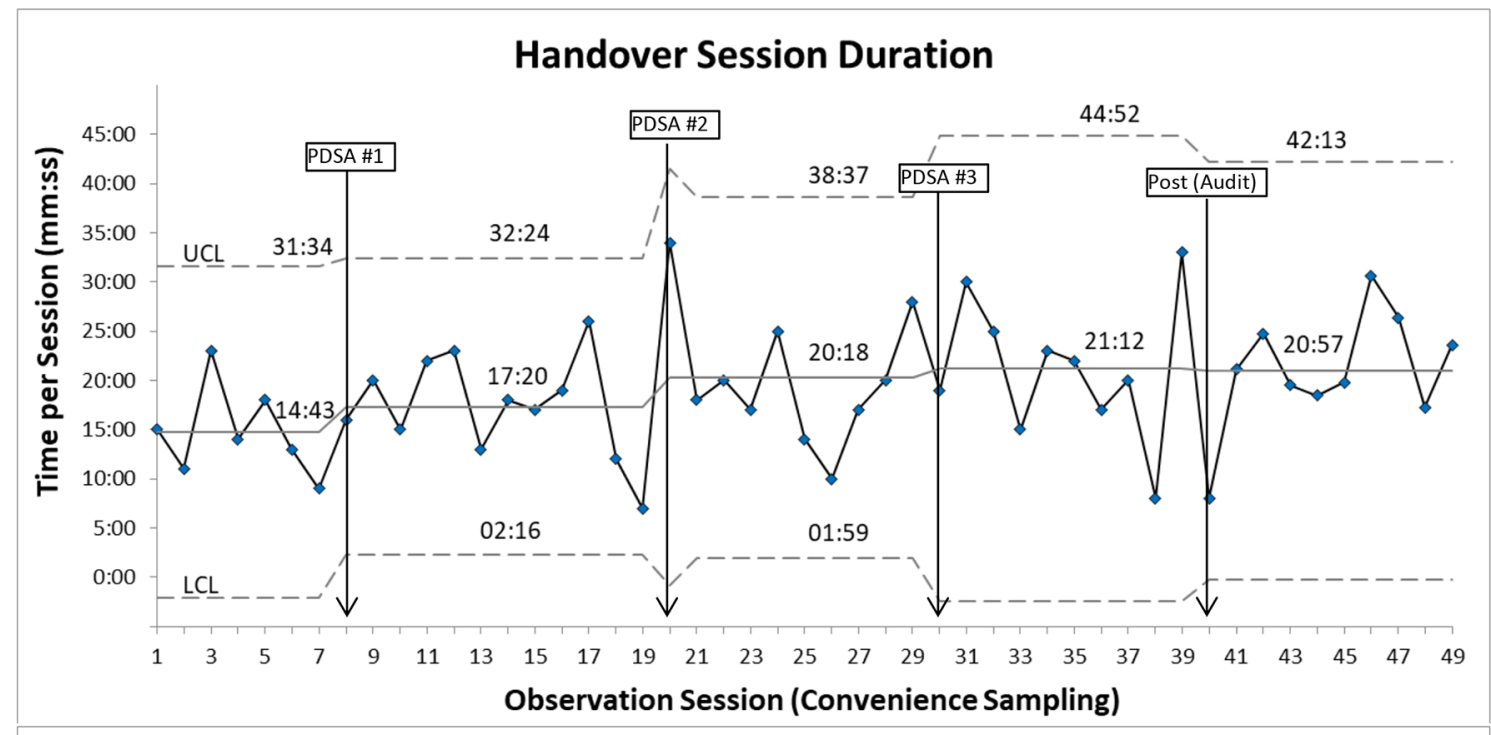

Handover Per-patient Duration*

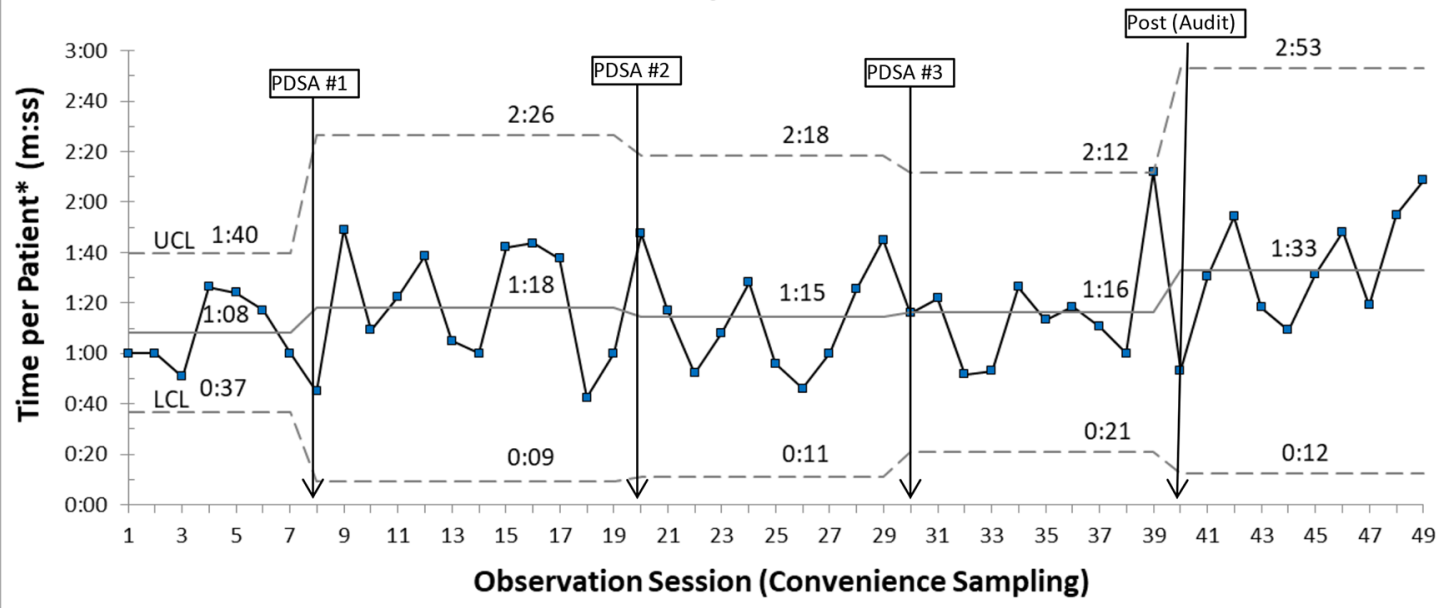

Figure 2 Duration of verbal handover; total time per session and mean time per patient. Note: calculation of per-patient duration excludes patients handed over by sticky note (e-note). PDSA, plan-do-study-act.

adjuncts, including cognitive support aids and 'bundling' of interventions, are critically important for successful and sustained improvements in handover quality. ${ }^{922} 24$ Cheung et alhighlighted the complex nature of ED handovers, and proposed that any frameworks or interventions to address this issue take into account the different stages of handover and local specific factors including physical space and workplace culture. ${ }^{1}$ Reflecting on the importance of taking local culture into account, our physician group adamantly rejected any change that involved filling out additional documentation. Using the acronym as a cognitive framework for handovers was an acceptable way of ensuring essential components were considered; the absence of a formal checklist did not seem to impact the success of our intervention.

Third, we leveraged previously published success factors for effective handovers. Frankel et al videotaped a series of resident-to-resident and nursing-to-nursing handovers and discovered several patterns of non-verbal handover behaviours, of which 'joint focus of attention' (defined as the individuals involved in the communication coordinating their verbal and visual attention jointly on an artefact such as a sign out tool, computer screen, or paper list) was deemed to have the greatest potential for increased quality and reliability. ${ }^{34}$ In our study, this took the form of reinforcing existing artefacts of handover such as the sticky note on the electronic whiteboard in our computer system.

An unexpected potential benefit that arose out of our implementation was how a structured handover framework enhanced resident education around this critical non-technical skill. Both staff and resident physicians reported the potential value of explicitly discussing and practicing the approach to preparing, delivering, and receiving handover information. Cate and Young have proposed creating explicit entrustable professional activity criteria for patient handovers ${ }^{35}$ and we feel that ED-specific frameworks such as ED-VITAL/ED-VSA may be good starting points for competency-based education efforts. 


\section{Limitations}

There were several limitations to our study. First, there are no validated measures for ED handover quality. We had to define adequacy of handovers based on the components deemed to be important in previous handover literature and by our local physician input; this method was used in a similar study in the paediatric ED population. ${ }^{30}$ The assumption in this study was that more consistent communication of these items would correlate with better quality of ED handovers. The definition of 'adequacy' used in our study (ie, $50 \%$ of components communicated) was made based on pragmatic expert opinion and experience; when we analysed our data using a cut-off of $100 \%$ of components communicated, only $21 \%$ of patients in the postimplementation audit period would have met that threshold. We believe this reflects our real-world experience in the ED, where the majority of patients being handed over often do not require minute details of all possible handover information categories in order for safe transfer of care. We did not have any robust adverseevent or regularly used patient safety tracking system in our ED at the time of the study, thus we were unable to collect direct clinical outcome data related to ED handovers.

Second, there was the potential for a Hawthorne effect whenever direct observations were conducted. We tried to mitigate this by having a QI coordinator who was not well known to the clinicians doing the direct observations (eg, 'fly on the wall' approach). There was also the potential for social desirability bias for survey participants. In addition, these surveys were not validated and were developed to suit our local needs.

Third, we were unable to collect more robust frontline physician feedback on the use of the new handover tool. We had designed paper surveys for the overnight physicians to complete at the end of their shift; however, the response rate was extremely poor. A number of logistical factors contributed to the extreme difficulty of getting clinicians to fill out such surveys, including physical and mental exhaustion of doing an overnight shift, lack of protected time during the shift to reflect and complete the survey, as well as overall survey fatigue in the group. We settled for in the moment direct feedback that was obtained through informal questioning by the QI coordinator immediately after observing handovers.

Finally, this was a single site study showcasing a handover tool that was heavily influenced by local preferences and nuances of existing handover culture. While this might limit generalisability of the tool itself, we believe this work highlights the importance of modifying any standardised tool to enhance adoption at the local context.

\section{CONCLUSIONS}

This paper described the development and implementation of an ED-specific handover tool using a multipronged approach. Success factors included assessment of readiness for change using mixed methods; engagement of frontline staff in development and feedback; incorporation of local preferences and context; and utilisation of formal QI methodology. Future work is needed to further examine how to incorporate ED handover tools into competency-based medical education. This customised approach to developing and implementing a standardised handover process can be used by any ED.

\section{Author affiliations}

${ }^{1}$ Department of Emergency Medicine, Ottawa Hospital, Ottawa, Ontario, Canada ${ }^{2}$ Department of Emergency Medicine, University of Ottawa, Ottawa, Ontario, Canada ${ }^{3}$ Department of Emergency Medicine, Ottawa Hospital Research Institute, Ottawa, Ontario, Canada

\section{Presented at}

ESHK, GC, SW, LAC. Development and implementation of a standardised ED handover tool (Moderated poster). Institute for Healthcare Improvement National Forum Scientific Symposium. Florida, OL USA. December 2018. Development and implementation of a standardised ED handover tool (Oral). Canadian Association of Emergency Physicians Annual Conference. Calgary AB, Canada. May 2018. Development and implementation of a standardised ED handover tool (Poster). Society of Academic Emergency Medicine Annual Conference. Indianapolis IN, USA. May 2018. Development and implementation of a standardised ED handover tool (Oral and Poster). The Ottawa Hospital 13th Annual Patient Safety Conference. Ottawa ON, Canada. October 2017.

Acknowledgements We wish to acknowledge the following key stakeholders who helped us develop and champion our handover framework: Dr Samantha CalderSprackman, Dr Stephen Choi, Dr Eric Clark, Dr Jason Frank, Dr Andrew Gee, Dr Guy Hebert, Dr George Mastoras, Dr Brandi Read, Dr Michael Woo, Cari Poulin RN.

Contributors ESHK and LAC conceived and planned the study. ESHK, SW, MA and LAC developed and revised the intervention. GC and SW performed direct observations and data collection. ESHK and GC analysed the data. All authors contributed in the write up and revision of the final manuscript.

Funding This work was supported by internal funding provided by the University of Ottawa Department of Emergency Medicine.

Competing interests None declared.

Patient consent for publication Not required.

Ethics approval According to the policy and standard operating procedures that govern research at the Ottawa Hospital Research Institute, this work met criteria for quality improvement activities exempt from full ethics review. All patient identifiers were removed from study data to protect privacy and confidentiality of patients; observation dates were replaced with session numbers to protect physician identity. Consent was implied by ED physicians by participating in the education, and physicians had the option to decline direct observation of their handovers.

Provenance and peer review Not commissioned; externally peer reviewed.

Data availability statement All data relevant to the study are included in the article or uploaded as supplementary information.

Open access This is an open access article distributed in accordance with the Creative Commons Attribution Non Commercial (CC BY-NC 4.0) license, which permits others to distribute, remix, adapt, build upon this work non-commercially, and license their derivative works on different terms, provided the original work is properly cited, appropriate credit is given, any changes made indicated, and the use is non-commercial. See: http://creativecommons.org/licenses/by-nc/4.0/.

ORCID iD

Edmund S H Kwok http://orcid.org/0000-0003-2339-1019

\section{REFERENCES}

1 Cheung DS, Kelly JJ, Beach C, et al. Improving handoffs in the emergency department. Ann Emerg Med 2010;55:171-80.

2 Kachalia A, Gandhi TK, Puopolo AL, et al. Missed and delayed diagnoses in the emergency department: a study of closed malpractice claims from 4 liability insurers. Ann Emerg Med 2007;49:196-205. 
3 Ye K, McD Taylor D, Knott JC, et al. Handover in the emergency department: deficiencies and adverse effects. Emerg Med Australas 2007;19:433-41.

4 Cook RI, Render M, Woods DD. Gaps in the continuity of care and progress on patient safety. BMJ 2000;320:791-4.

5 Horwitz LI, Moin T, Krumholz HM, et al. Consequences of inadequate sign-out for patient care. Arch Intern Med 2008;168:1755-60.

6 Sinha M, Shriki J, Salness R, et al. Need for Standardized Sign-out in the Emergency Department: A Survey of Emergency Medicine Residency and Pediatric Emergency Medicine Fellowship Program Directors. Acad Emerg Med 2007;14:192-6.

7 Kwok ES, White S, Calder LA. P070: improving handovers in the emergency department: implementation of a standardized team approach. CJEM 2016;18:S101-2.

8 Calder LA, Haligua A, Frank JR, et al. Handover in the emergency department: an observational analysis of quality and elements. CJEM 2012;14:S22.

9 Starmer AJ, Spector ND, Srivastava R, et al. Changes in medical errors after implementation of a handoff program. N Engl J Med 2014;371:1803-12.

10 Fernando K, Adshead N, Dev S, et al. Emergency department multiprofessional handover. Clin Teach 2013;10:219-23.

11 The Joint Commission. Sentinel event alert issue 58: inadequate hand-off communication, 2017. Available: www.jointcommission. org/sentinel_event_alert_58_inadequate_handoff_communications/ [Accessed 23 May 2019].

12 Abraham J, Kannampallil T, Patel VL. A systematic review of the literature on the evaluation of handoff tools: implications for research and practice. J Am Med Inform Assoc 2014;21:154-62.

13 Cwinn MA, Forster AJ, Cwinn AA, et al. Prevalence of information gaps for seniors transferred from nursing homes to the emergency department. CJEM 2009;11:462-72.

14 Smith C, Buzalko R, Anderson N, et al. Evaluation of a novel handoff communication strategy for patients admitted from the emergency department. WestJEM 2018;19:372-9.

15 Smith CJ, Britigan DH, Lyden E, et al. Interunit handoffs from emergency department to inpatient care: a cross-sectional survey of physicians at a university medical center. J. Hosp. Med. 2015;10:711-7.

16 Meisel ZF, Shea JA, Peacock NJ, et al. Optimizing the patient handoff between emergency medical services and the emergency department. Ann Emerg Med 2015;65:310-7.

17 Gonzalo JD, Yang JJ, Stuckey HL, et al. Patient care transitions from the emergency department to the medicine ward: evaluation of a standardized electronic signout tool. Int J Qual Health Care 2014;26:337-47.

18 Farhan M, Brown R, Woloshynowych M, et al. The ABC of handover: a qualitative study to develop a new tool for handover in the emergency department. Emerg Med J 2012;29:941-6.
19 Farhan M, Brown R, Vincent C, et al. The ABC of handover: impact on shift handover in the emergency department. Emerg Med $\mathrm{J}$ 2012;29:947-53.

20 Williams S, Chien J, Ribeira R. Ed I-PASS: a streamlined version of the I=PASS patient handoff tool for the emergency department. JETem 2017;2:L1-13.

21 Rüdiger-Stürchler M, Keller DI, Bingisser R. Emergency physician intershift handover - can a dINAMO checklist speed it up and improve quality? Swiss Med Wkly 2010;140:w13085.

22 Abele J, Fuller D, Kerr M, et al. New tool aims to standardize handoffs in the ED, boosting safety and preventing communication failures. ED Manag 2013;25:85-96.

23 Dubosh NM, Carney D, Fisher J, et al. Implementation of an emergency department sign-out checklist improves transfer of information at shift change. J Emerg Med 2014;47:580-5.

24 Gillet A, Ghuysen A, Bonhomme S, et al. Cognitive support for a better handoff: does it improve the quality of medical communication at shift change in an emergency department? Eur J Emerg Med 2015;22:192-8

25 Mullan PC, Macias CG, Hsu D, et al. A novel briefing checklist at shift handoff in an emergency department improves situational awareness and safety event identification. Pediatr Emerg Care 2015;31:231-8.

26 Clay-Williams R, Colligan L. Back to basics: checklists in aviation and healthcare. BMJ Qual Saf 2015;24:428-31.

27 Reames BN, Scally CP, Thumma JR, et al. Evaluation of the effectiveness of a surgical checklist in Medicare patients. Med Care 2015;53:87-94

28 Urbach DR, Govindarajan A, Saskin R, et al. Introduction of surgical safety checklists in Ontario, Canada. N Engl J Med 2014;370:1029-38.

29 Maughan BC, Lei L, Cydulka RK. Ed handoffs: observed practices and communication errors. Am J Emerg Med 2011;29:502-11.

30 Gopwani PR, Brown KM, Quinn MJ, et al. Sound: a structured handoff tool improves patient handoffs in a pediatric emergency department. Pediatr Emerg Care 2015;31:83-7.

31 Heilman J, Flanigan M, Nelson A, et al. Adapting the I-PASS handoff program for emergency department inter-shift handoffs. WestJEM 2016;17:756-61.

32 Kicken W, Van der Klink M, Barach P, et al. Handover training: does one size fit all? the merits of mass customisation. BMJ Qual Saf 2012;21:i84-8.

33 Blyth C, Bost N, Shiels S. Impact of an education session on clinical handover between medical shifts in an emergency department: a pilot study. Emerg Med Australas 2017;29:336-41.

34 Frankel RM, Flanagan M, Ebright P, et al. Context, culture and (non-verbal) communication affect handover quality. BMJ Qual Saf 2012;21:i121-8.

35 ten Cate O, Young JQ. The patient handover as an entrustable professional activity: adding meaning in teaching and practice. BMJ Qual Saf 2012;21:i9-12. 